\title{
CA19-9-Low\&Lewis (+) pancreatic cancer: a unique subtype
}

Guopei Luo $^{\text {a,b,c }}$, Chen Liu ${ }^{\text {a,b,c }}$, Meng Guo, ${ }^{\text {a,b,c }}$, Jiang Long ${ }^{\text {a,b,c }}$, Zuqiang Liu ${ }^{\text {a,b,c }}$, Zhiwen Xiao $^{\text {a,b,c }}$, Kaizhou Jin ${ }^{\text {a,b,c }}$, He Cheng ${ }^{\text {a,b,c }}$, Yu Lu ${ }^{\text {a,b,c }}$, Quanxing Ni ${ }^{\text {a,b,c }}$, Xianjun Yu*, $\mathrm{a}, \mathrm{b}, \mathrm{c}$ 


\section{ABSTRACT}

The study was performed to identify unique subtype from the long-term survival (> 24 months) patients with advanced pancreatic cancer (1039 cases). Long-term survival patients had higher proportion of low secretion of carbohydrate antigen 19-9 (CA19-9) than that of patients with non long-term survival $(\mathrm{P}<0.001)$. Considering the impact of Lewis status on CA19-9 secretion, Lewis genotypes were further determined by Sanger sequencing. The prognosis of CA19-9-Low\&Lewis (-) patients was worse than that of CA19-9-Low\&Lewis $(+)$ (hazard ratio $(\mathrm{HR})=0.37, \mathrm{P}<0.001)$. The proportion of epidermal growth factor receptor (EGFR) (-) cases was lower in the CA19-9-Low\&Lewis $(+)$ subgroup than that in other patients $(P=0.047)$. For the CA19-9-Low\&Lewis (+) subgroup, chemotherapy plus radiotherapy but not chemotherapy was found to be an independent prognostic factor (versus best supportive care, chemotherapy, $\mathrm{HR}=0.71, \mathrm{P}=0.267$; chemotherapy plus radiotherapy, $\mathrm{HR}=0.33, \mathrm{P}=0.022)$. We conclude that CA19-9-Low\&Lewis $(+)$ pancreatic cancer is a unique subtype with special biological properties.

Keywords: Pancreatic adenocarcinoma; Lewis antigen; heterogeneity; CA19-9 


\section{Introduction}

Pancreatic cancer is a dismal malignancy and patients often survive less than one year [1-3]. Nevertheless, a minority of patients with pancreatic cancer have shown long-term survival [4]. It has been documented that pancreatic cancer is comprised of highly heterogeneous tumors, with certain subgroups possessing different biological properties [5]. Patients who show long-term survival might be a unique subgroup whose tumors harbor special biological and clinical features, especially for patients with advanced pancreatic cancer [4].

CA19-9 is currently the most important biomarker for pancreatic cancer [6, 7]. The recommended upper limit of CA19-9 as a biomarker for pancreatic cancer was 37 $\mathrm{U} / \mathrm{mL}[8,9]$. Approximately $20 \%$ of patients with pancreatic cancers have no or low secretion of CA19-9. The Lewis antigen system is a human blood group system and the Lewis (-) individuals, accounting for approximately $5 \%$ to $10 \%$ of the population, have been confirmed to have no or minimal secretion of CA19-9 $[8,10]$. The following variants in the Lewis gene: T59G, T202C, C314T, G508A, and T1067A, have been reported to determine Lewis genotypes [11]. Our previous study has showed that the prognosis of Lewis (-) patients with advanced pancreatic cancer was worse than that of Lewis (+) patients [11]. However, several studies have found that pancreatic cancer patients with a normal range of CA19-9 levels $(\leq 37 \mathrm{U} / \mathrm{mL})$ had a better prognosis than patients with high levels of CA19-9 (> $37 \mathrm{U} / \mathrm{mL})$ [12-14]. These observations indicate that pancreatic cancer patients with low-CA19-9 secretion and Lewis (+) may be long-term survivals with unique features. 
In the study, unique subgroup was identified from the long-term survival patients with advanced pancreatic cancer. Molecular features of the identified subgroup were examined. The efficacy of major treatments including chemotherapy and radiotherapy were analyzed in the identified subgroup.

\section{Methods}

\subsection{Patients}

Medical records of patients with advanced pancreatic cancer were retrieved from a prospectively maintained database by the Fudan University Shanghai Cancer Center. Tumor stages were classified using the $7^{\text {th }}$ edition of the American Joint Committee on Cancer (AJCC) staging classification and advanced pancreatic cancer was defined as stage III, IV disease [15]. All diagnoses were based on cytological or histological evidence of adenocarcinoma of the pancreas. Pancreatic endocrine tumors, intraductal papillary mucinous neoplasm associated pancreatic adenocarcinoma, cystoadenocarcinoma, or acinar carcinomas were excluded. Patients lacking detailed information about major treatments, or information of Lewis status, or baseline serum CA19-9 levels, were also excluded. Long-term survival patients were defined as patients survived longer than 24 months after the final diagnosis. For the analysis of therapeutic efficacy, patients underwent Gemcitabine-based chemotherapy, patients underwent radiotherapy that was considered to affect prognosis, and patients with best supportive care were included. The study protocol was approved by the local ethics commission. Written informed consent was obtained from all of the participants in this study. 


\subsection{Serum assays for CA19-9}

Baseline serum CA19-9 levels were examined within two weeks before the initiation of major treatment as previously described [16]. Serum CA19-9 were detected using an electrochemiluminescence immunoassay on the Roche Cobas e601 (Roche MODU D + P model, D2400-P800) immunoassay analyzer (Roche Diagnostics, Mannheim, Germany). The recommended upper limit of CA19-9 as a diagnostic biomarker for pancreatic cancer was $37 \mathrm{U} / \mathrm{mL}[8,9]$. CA19-9-Low was defined as CA19-9 level lower than $37 \mathrm{U} / \mathrm{mL}$.

\subsection{Sequencing for Lewis gene}

Lewis status was determined on the basis of the Lewis genotypes by Sanger sequencing using genomic DNA [11]. Lewis (-) genotypes were measured on the basis of the following variants in the Lewis gene: T59G, T202C, C314T, G508A, and T1067A [11]. Blood samples were collected and preserved before initiating major treatments by the Central Tissue Bank. Genomic DNA was extracted from the blood samples on the basis of standard protocols. PCR reaction was amplified using the

following primers (358F, GGGTGCAGCCAAGCCACAA, 358R, AGGTGGGAGGCGTGACTTAGG; P1F, ACTTGGAGCCACCCCCTAACTGCCA, 508R, CGGCCTCTCAGGTGAACCAAGCCGCT). The amplified products were examined by a 3730XL DNA Sequencer (Applied Biosystems, USA).

\subsection{Immunohistochemical analysis}

Immunohistochemical analysis of the formalin-fixed, paraffin-embedded (FFPE) tumor tissues for TP53, SMAD4, CDKN2A, EGFR, and HER2 expressions was 
examined using the Dako EGFR PharmDx TM kit (Dako) [17]. The FFPE specimens were measured for immunostaining by two independent pathologists blinded to the clinical information (recorded at Fudan University Shanghai Cancer Center). Patients were divided into positive and negative subgroups according to the intensity and completeness of immunohistochemical staining.

\subsection{Statistical Analyses}

The primary end point was overall survival with the follow-up information updated in March 2016. The survival period was determined from the date of final diagnosis to the date of death or censored at the latest follow-up. Survival analysis was performed by the Kaplan-Meier estimates with the STATA 12.0 statistical software package (StataCorp LP, College Station, TX, USA). Different arms were compared using Log-rank tests. Univariate and multivariate survival analyses for overall survival were performed by Cox proportional hazards models. Continuous variables were examined using Student's t-test or the Rank-sum test where appropriate. Dichotomous variables were examined by the $\chi^{2}$ test or the Fisher exact test where appropriate. Two-sided $p$ values $<0.05$ were viewed statistically significant.

\section{Results}

3.1. High proportion of CA19-9 Low-secretion in long-term survival patients with advanced pancreatic cancer

In the study, 1039 patients with advanced pancreatic cancer were included, with a median survival time of 7.6 months. Twenty-eight (2.7\%) patients survived more than 24 months. Table 1 summarized clinicopathologic characteristics of long-term 
survival patients. Besides age $\leq 60$ years $(\mathrm{P}=0.034)$ and stage III diseases $(\mathrm{P}=0.012)$, long-term survival patients had higher proportion of radiotherapy $(\mathrm{P}<0.001)$ and low secretion of CA19-9 (CA19-9-Low, $\mathrm{P}<0.001$ ) than that of patients with non long-term survival. Theses results suggest that CA19-9-Low pancreatic cancer may be a unique subgroup and it may have well response to radiotherapy. The superior prognosis of CA19-9-Low patients was further demonstrated by Kaplan-Meier curves in Fig. 1A $(\mathrm{P}<0.001)$.

\subsection{Superior prognosis of Lewis $(+)$ patients in the CA19-9-Low subgroup}

Lewis negative individuals are well-known for the low-secretion of CA19-9 [8, 10]. Therefore, the Lewis genotypes of CA19-9-Low patients with advanced pancreatic cancer were determined by Sanger sequencing. In the CA19-9-Low subgroup, 68 $(68 / 192,35.4 \%)$ cases were confirmed to be Lewis (-) and $124(124 / 192,64.6 \%)$ cases were Lewis (+). The prognosis of CA19-9-Low\&Lewis (-) patients was worse than that of CA19-9-Low\&Lewis $(+)(\mathrm{HR}=0.37, \mathrm{P}<0.001$, Fig. 1B). CA19-9-Low\&Lewis (+) was further found to be an independent prognostic factor in patients with advanced pancreatic cancer by multivariate analyses $(\mathrm{HR}=0.52, \mathrm{P}<$ 0.001, Table 2), indicating that CA19-9-Low\&Lewis (+) patients is a unique subtype with less aggressive properties.

\subsection{Molecular features of CA19-9-Low \&Lewis (+) pancreatic cancer}

In order to examine whether the CA19-9-Low\&Lewis (+) subgroup harbors unique molecular profiles, TP53, SMAD4, CDKN2A, EGFR, and HER2 expression were examined by immunohistochemistry. The proportion of EGFR (-) cases was lower in 
the CA19-9-Low\&Lewis (+) subgroup than that in the non CA19-9-Low\&Lewis (+) subgroup ( $\mathrm{P}=0.047$, Table 3). However, no statistical difference was found between the CA19-9-Low\&Lewis (+) and the non CA19-9-Low\&Lewis (+) subgroups in TP53, SMAD4, CDKN2A, and HER2 expression.

\subsection{Efficacy of major treatments for the CA19-9-Low\&Lewis (+) subgroup}

Patients with advanced pancreatic cancer were included in the analysis. For patients underwent chemotherapy, only patients underwent Gemcitabine-based chemotherapy were included. For patients underwent radiotherapy, it was delivered to the primary tumor and/or major metastases that were thought to affect prognosis. For the non CA19-9-Low\&Lewis (+) subgroup, chemotherapy and chemotherapy plus radiotherapy were found to be independent prognostic factors (versus best supportive care, chemotherapy, $\mathrm{HR}=0.57,95 \% \mathrm{CI}, 0.46-0.70, \mathrm{P}<0.001$; chemotherapy plus radiotherapy, $\mathrm{HR}=0.47,95 \% \mathrm{CI}, 0.32-0.68, \mathrm{P}<0.001)$. For the CA19-9-Low\&Lewis (+) subgroup, chemotherapy plus radiotherapy but not chemotherapy was found to be an independent prognostic factor (versus best supportive care, chemotherapy, HR = $0.71,95 \% \mathrm{CI}, 0.39-1.30, \mathrm{P}=0.267$; chemotherapy plus radiotherapy, $\mathrm{HR}=0.33,95 \%$ CI, 0.13-0.85, $\mathrm{P}=0.022$ ). Similar results were shown by Kaplan-Meier curves in Figure 2. Statistical difference in survival was observed for different tumor stages of patients with CA19-9-Low\&Lewis (+) pancreatic cancer (median survival time, stage I, II, 25.9 months; stage III, 18.8 months; stage IV, 10.1 months; $\mathrm{P}<0.001$ by Log-rank test), indicating that tumor stage has a prognostic role in patients with CA19-9-Low\&Lewis (+) pancreatic cancer. In addition, median survival time of 
patients with stage III CA19-9-Low\&Lewis (+) pancreatic cancer (18.8 months) was even longer than patients with stage I, II non CA19-9-Low\&Lewis (+) pancreatic cancer (16.6 months), suggesting that a modified staging system should be proposed for CA19-9-Low\&Lewis (+) pancreatic cancer.

\section{Discussion}

Several studies have found that pancreatic cancer patients with a normal range of CA19-9 levels $(\leq 37 \mathrm{U} / \mathrm{mL})$ had a better prognosis than patients with high levels of CA19-9 (> $37 \mathrm{U} / \mathrm{mL}$ ) [12-14]. For example, Hartwig et al [12] found that the prognosis of CA19-9 non-secretors (CA19-9 $<5 \mathrm{U} / \mathrm{ml}$, taken as Lewis (-)) was comparable to those who had a normal range of CA19-9 levels $(5-37 \mathrm{U} / \mathrm{mL})$ and all these patients had better prognosis than patients with high levels of CA19-9 $(\geq 37$ $\mathrm{U} / \mathrm{mL}$ ). However, these studies did not determine the Lewis antigen status [12-14]. In this study, by determining Lewis status by sequencing, CA19-9-Low\&Lewis (+) subtype was found to have superior prognosis compared with other patients for advanced pancreatic cancer. It is worth to mention that the less aggressiveness of CA19-9-Low\&Lewis (+) pancreatic cancer cannot be attributed to early stages but biological properties because only patients with advanced pancreatic cancer were included in the analyses. In addition, no difference was found between CA19-9-Low\&Lewis (+) pancreatic cancers and other pancreatic cancers in the expression of three major genes (TP53, SMAD4, CDKN2A), which was similar to a previous report [18] . 
Several studies have found that patients with normal CA19-9 have different efficacy to different chemotherapy regimen. Von Hoff et al [19] found that patients with normal CA19-9 had poor therapeutic response to nab-Paclitaxel plus Gemcitabine (nab-Paclitaxel plus Gemcitabine versus Gemcitabine alone, normal, HR $=1.07 ;<59 \times$ upper limit of the normal range $(\mathrm{ULN}), \mathrm{HR}=0.83 ; \geq 59 \times \mathrm{ULN}, \mathrm{HR}$ $=0.61$ ). In another study, Conroy et al [20] showed that patients with normal CA19-9 had well response to oxaliplatin, irinotecan, leucovorin, fluorouracil (FOLFIRINOX) (FOLFIRINOX versus Gemcitabine alone, normal, $\mathrm{HR}=0.42$; abnormal, $\mathrm{HR}=0.58$ ). In our previous study, chemotherapy had a significant survival benefit in the EGFR (+) subgroup $(\mathrm{P}=0.002)$, and radiotherapy was highly associated with increased postoperative survival in the EGFR $(-)$ subgroup $(\mathrm{P}=0.029)$ [17]. Uesaka et al [21] assigned patients with resected pancreatic cancer to receive S-1 (CA19-9 normal in $81 \%$ of cases) or Gemcitabine (CA19-9 normal in $77 \%$ of cases) and the median overall survival was 46.5 months with S-1 and 25.5 months with Gemcitabine. In this study, the CA19-9-Low\&Lewis (+) subgroup had lower proportion of EGFR expression than the non CA19-9-Low\&Lewis (+) subgroup. Moreover, the CA19-9-Low\&Lewis (+) subgroup had well response to radiotherapy and poor response to Gemcitabine-based chemotherapy. According to previous studies $[17,19$, 20] and our current results, we hypothesized that CA19-9-Low\&Lewis (+) pancreatic cancer may benefit from fluorouracil based chemotherapy or radiotherapy and CA19-9 abnormal pancreatic cancer may be sensitive to Gemcitabine based chemotherapy. In addition, chemotherapy plus radiotherapy would be an especially 
beneficial treatment for CA19-9-Low/Lewis (+) pancreatic cancer. However, further prospective clinical trials are needed to confirm our hypothesis.

The molecular mechanisms of the low secretion of CA19-9 in the CA19-9-Low subtype are largely undefined. The CA19-9-Low subtype had a low level of neutrophil-lymphocyte ratio, an important indicator of systemic inflammation [22]. The inflammatory microenvironment in pancreatic cancer can regulate the expression of tumor-associated sialylated antigens [23]. In addition, the CA19-9-Low subtype had low levels of fasting plasma glucose and previous study has found a positive correlation between CA19-9 and fasting plasma glucose [24]. Moreover, a Lewis gene heterozygous mutation could reduce the fucosyltransferase activity in saliva and decrease the secretion of CA19-9 in serum [25].

\section{Conflict of Interest}

There is no conflict of interest.

\section{Acknowledgements}

This study was jointly funded by the National Science Foundation for

Distinguished Young Scholars of China (No. 81625016), the National Natural Science Foundation of China (No. 81172276) and the Prospective Clinical Trial Project, Shanghai Cancer Center (YJLC201403). We thank Prof. Dong Xiang from Shanghai Blood Center, and Prof. Menghong Sun from Tissue Bank, Shanghai Cancer Center for the technical assistance. 


\section{References}

[1] D.P. Ryan, T.S. Hong, N. Bardeesy, Pancreatic adenocarcinoma, N Engl J Med, 371 (2014) 1039-1049.

[2] R.L. Siegel, K.D. Miller, A. Jemal, Cancer statistics, 2015, CA Cancer J Clin, 65 (2015) 5-29.

[3] J. Long, G.P. Luo, Z.W. Xiao, Z.Q. Liu, M. Guo, L. Liu, C. Liu, J. Xu, Y.T. Gao, Y. Zheng, C. Wu, Q.X. Ni, M. Li, X. Yu, Cancer statistics: current diagnosis and treatment of pancreatic cancer in Shanghai, China, Cancer Lett, 346 (2014) 273-277.

[4] C.R. Ferrone, R. Pieretti-Vanmarcke, J.P. Bloom, H. Zheng, J. Szymonifka, J.A. Wargo, S.P. Thayer, G.Y. Lauwers, V. Deshpande, M. Mino-Kenudson, C. Fernandez-del Castillo, K.D. Lillemoe, A.L. Warshaw, Pancreatic ductal adenocarcinoma: long-term survival does not equal cure, Surgery, 152 (2012) S43-49.

[5] N. Waddell, M. Pajic, A.M. Patch, D.K. Chang, K.S. Kassahn, P. Bailey, A.L. Johns, D. Miller, K. Nones, K. Quek, M.C. Quinn, A.J. Robertson, M.Z. Fadlullah, T.J. Bruxner, A.N. Christ, I. Harliwong, S. Idrisoglu, S. Manning, C. Nourse, E. Nourbakhsh, S. Wani, P.J. Wilson, E. Markham, N. Cloonan, M.J. Anderson, J.L. Fink, O. Holmes, S.H. Kazakoff, C. Leonard, F. Newell, B. Poudel, S. Song, D. Taylor, S. Wood, Q. Xu, J. Wu, M. Pinese, M.J. Cowley, H.C. Lee, M.D. Jones, A.M. Nagrial, J. Humphris, L.A. Chantrill, V. Chin, A.M. Steinmann, A. Mawson, E.S. Humphrey, E.K. Colvin, A. Chou, C.J. Scarlett, 
A.V. Pinho, M. Giry-Laterriere, I. Rooman, J.S. Samra, J.G. Kench, J.A. Pettitt, N.D. Merrett, C. Toon, K. Epari, N.Q. Nguyen, A. Barbour, N. Zeps, N.B. Jamieson, J.S. Graham, S.P. Niclou, R. Bjerkvig, R. Grutzmann, D. Aust, R.H. Hruban, A. Maitra, C.A. Iacobuzio-Donahue, C.L. Wolfgang, R.A. Morgan, R.T. Lawlor, V. Corbo, C. Bassi, M. Falconi, G. Zamboni, G. Tortora, M.A. Tempero, A.J. Gill, J.R. Eshleman, C. Pilarsky, A. Scarpa, E.A. Musgrove, J.V. Pearson, A.V. Biankin, S.M. Grimmond, Whole genomes redefine the mutational landscape of pancreatic cancer, Nature, 518 (2015) 495-501.

[6] J.L. Humphris, D.K. Chang, A.L. Johns, C.J. Scarlett, M. Pajic, M.D. Jones, E.K. Colvin, A. Nagrial, V.T. Chin, L.A. Chantrill, J.S. Samra, A.J. Gill, J.G. Kench, N.D. Merrett, A. Das, E.A. Musgrove, R.L. Sutherland, A.V. Biankin, The prognostic and predictive value of serum CA19.9 in pancreatic cancer, Ann Oncol, 23 (2012) 1713-1722.

[7] A.C. Berger, M. Garcia, Jr., J.P. Hoffman, W.F. Regine, R.A. Abrams, H. Safran, A. Konski, A.B. Benson, 3rd, J. MacDonald, C.G. Willett, Postresection CA 19-9 predicts overall survival in patients with pancreatic cancer treated with adjuvant chemoradiation: a prospective validation by RTOG 9704, J Clin Oncol, 26 (2008) 5918-5922.

[8] M.A. Tempero, E. Uchida, H. Takasaki, D.A. Burnett, Z. Steplewski, P.M. Pour, Relationship of carbohydrate antigen 19-9 and Lewis antigens in pancreatic cancer, Cancer Res, 47 (1987) 5501-5503.

[9] F. Safi, H.G. Beger, R. Bittner, M. Buchler, W. Krautzberger, CA 19-9 and 
pancreatic adenocarcinoma, Cancer, 57 (1986) 779-783.

[10] P.M. Pour, M.M. Tempero, H. Takasaki, E. Uchida, Y. Takiyama, D.A. Burnett, Z. Steplewski, Expression of blood group-related antigens ABH, Lewis A, Lewis B, Lewis $\mathrm{X}$, Lewis $\mathrm{Y}$, and CA 19-9 in pancreatic cancer cells in comparison with the patient's blood group type, Cancer Res, 48 (1988) $5422-5426$.

[11] Luo G, Liu C, Guo M, Cheng H, Lu Y, Jin K, Liu L, Long J, Xu J, Lu R, Ni Q, Yu X. Potential biomarkers in Lewis negative patients with pancreatic cancer. Annals of Surgery. 2016. [Epub ahead of print].

[12] W. Hartwig, O. Strobel, U. Hinz, S. Fritz, T. Hackert, C. Roth, M.W. Buchler, J. Werner, CA19-9 in potentially resectable pancreatic cancer: perspective to adjust surgical and perioperative therapy, Ann Surg Oncol, 20 (2013) 2188-2196.

[13] A.C. Berger, I.M. Meszoely, E.A. Ross, J.C. Watson, J.P. Hoffman, Undetectable preoperative levels of serum CA 19-9 correlate with improved survival for patients with resectable pancreatic adenocarcinoma, Ann Surg Oncol, 11 (2004) 644-649.

[14] L.K. Martin, L. Wei, E. Trolli, T. Bekaii-Saab, Elevated baseline CA19-9 levels correlate with adverse prognosis in patients with early- or advanced-stage pancreas cancer, Med Oncol, 29 (2012) 3101-3107.

[15] S.B. Edge, C.C. Compton, The American Joint Committee on Cancer: the 7th edition of the AJCC cancer staging manual and the future of TNM, Ann Surg 
Oncol, 17 (2010) 1471-1474.

[16] G. Luo, Z. Xiao, J. Long, Z. Liu, L. Liu, C. Liu, J. Xu, Q. Ni, X. Yu, CA125 is superior to CA19-9 in predicting the resectability of pancreatic cancer, J Gastrointest Surg, 17 (2013) 2092-2098.

[17] M. Guo, G. Luo, C. Liu, H. Cheng, Y. Lu, K. Jin, Z. Liu, J. Long, L. Liu, J. Xu, D. Huang, Q. Ni, X. Yu, The Prognostic and Predictive Role of Epidermal Growth Factor Receptor in Surgical Resected Pancreatic Cancer, Int J Mol Sci, 17 (2016).

[18] M. Dal Molin, M. Zhang, R.F. de Wilde, N.A. Ottenhof, N. Rezaee, C.L. Wolfgang, A. Blackford, B. Vogelstein, K.W. Kinzler, N. Papadopoulos, R.H. Hruban, A. Maitra, L.D. Wood, Very Long-term Survival Following Resection for Pancreatic Cancer Is Not Explained by Commonly Mutated Genes: Results of Whole-Exome Sequencing Analysis, Clin Cancer Res, 21 (2015) 1944-1950.

[19] D.D. Von Hoff, T. Ervin, F.P. Arena, E.G. Chiorean, J. Infante, M. Moore, T. Seay, S.A. Tjulandin, W.W. Ma, M.N. Saleh, M. Harris, M. Reni, S. Dowden, D. Laheru, N. Bahary, R.K. Ramanathan, J. Tabernero, M. Hidalgo, D. Goldstein, E. Van Cutsem, X. Wei, J. Iglesias, M.F. Renschler, Increased survival in pancreatic cancer with nab-paclitaxel plus gemcitabine, N Engl J Med, 369 (2013) 1691-1703.

[20] T. Conroy, F. Desseigne, M. Ychou, O. Bouche, R. Guimbaud, Y. Becouarn, A. Adenis, J.L. Raoul, S. Gourgou-Bourgade, C. de la Fouchardiere, J. Bennouna, J.B. Bachet, F. Khemissa-Akouz, D. Pere-Verge, C. Delbaldo, E. Assenat, B. 
Chauffert, P. Michel, C. Montoto-Grillot, M. Ducreux, FOLFIRINOX versus gemcitabine for metastatic pancreatic cancer, N Engl J Med, 364 (2011) $1817-1825$.

[21] K. Uesaka, N. Boku, A. Fukutomi, Y. Okamura, M. Konishi, I. Matsumoto, Y. Kaneoka, Y. Shimizu, S. Nakamori, H. Sakamoto, S. Morinaga, O. Kainuma, K. Imai, N. Sata, S. Hishinuma, H. Ojima, R. Yamaguchi, S. Hirano, T. Sudo, Y. Ohashi, Adjuvant chemotherapy of S-1 versus gemcitabine for resected pancreatic cancer: a phase 3, open-label, randomised, non-inferiority trial (JASPAC 01), Lancet, 388 (2016) 248-257.

[22] A. Akar, G.l. D, Z. Erdem, P. Sarathchandra, C. Tysoe, M. Pope, Acrogeric Ehlers-Danlos syndrome type IV: report of a new patient with additional findings, Eur J Dermatol, 12 (2002) 428-431.

[23] S. Bassaganas, H. Allende, L. Cobler, M.R. Ortiz, E. Llop, C. de Bolos, R. Peracaula, Inflammatory cytokines regulate the expression of glycosyltransferases involved in the biosynthesis of tumor-associated sialylated glycans in pancreatic cancer cell lines, Cytokine, 75 (2015) 197-206.

[24] S.H. Kim, C.O. Baek, K.A. Lee, T.S. Park, H.S. Baek, H.Y. Jin, Clinical implication of elevated CA 19-9 level and the relationship with glucose control state in patients with type 2 diabetes, Endocrine, 46 (2014) 249-255.

[25] T.F. Orntoft, E.M. Vestergaard, E. Holmes, J.S. Jakobsen, N. Grunnet, M. Mortensen, P. Johnson, P. Bross, N. Gregersen, K. Skorstengaard, U.B. Jensen, L. Bolund, H. Wolf, Influence of Lewis alpha1-3/4-L-fucosyltransferase (FUT3) 
gene mutations on enzyme activity, erythrocyte phenotyping, and circulating tumor marker sialyl-Lewis a levels, J Biol Chem, 271 (1996) 32260-32268. 


\section{Figure Legends}

Fig. 1. Kaplan-Meier curves showed survival of patients with advanced pancreatic cancer divided by CA19-9 and Lewis status. Patients with CA19-9-Low (CA19-9 $\leq$ $37 \mathrm{U} / \mathrm{mL}$ ) had better prognosis than patients with CA19-9-High (CA19-9 > $37 \mathrm{U} / \mathrm{mL}$ ) (A). For patients with CA19-9 low-secretion, Lewis (+) patients had better prognosis than Lewis (-) patients (B).

Fig. 2. Kaplan-Meier curves showed survival of patients with advanced pancreatic cancer divided by major treatments. For the non CA19-9-Low\&Lewis (+) subgroup, patients underwent chemotherapy or chemotherapy plus radiotherapy had better prognosis than patients had best supportive care (A). For the CA19-9-Low\&Lewis (+) subgroup, patients underwent chemotherapy plus radiotherapy had better prognosis than patients had best supportive care (B). However, no statistical significance was found between patients underwent chemotherapy and patients had best supportive care in the CA19-9-Low\&Lewis (+) subgroup (B) (Note: Chemo., chemotherapy; Radio., radiotherapy). 
Table 1

Characteristics of long-term survival patients with advanced pancreatic cancer.

\begin{tabular}{|c|c|c|c|c|}
\hline Characteristics & Items & Long-term survival & Non-long-term survival & $P$ value \\
\hline \multirow{2}{*}{ Age (year) } & $\leq 60$ & 18 & 446 & 0.034 \\
\hline & $>60$ & 10 & 565 & \\
\hline \multirow[b]{2}{*}{ Gender } & Male & 20 & 642 & 0.389 \\
\hline & $\mathrm{e}^{\text {Femal }}$ & 8 & 369 & \\
\hline \multirow{2}{*}{ Location } & Head & 11 & 382 & 0.872 \\
\hline & Others & 17 & 629 & \\
\hline \multirow{2}{*}{ Size $(\mathrm{cm})^{1}$} & $\leq 4$ & 14 & 439 & 0.476 \\
\hline & $>4$ & 13 & 538 & \\
\hline \multirow{2}{*}{ Stage } & III & 15 & 316 & 0.012 \\
\hline & IV & 13 & 695 & \\
\hline \multirow{2}{*}{ Chemotherapy } & Yes & 24 & 779 & 0.281 \\
\hline & No & 4 & 232 & \\
\hline \multirow{2}{*}{ Radiotherapy } & Yes & 9 & 82 & $<0.001$ \\
\hline & No & 19 & 929 & \\
\hline \multirow{2}{*}{ CA19-9 (U/mL) } & $\leq 37$ & 15 & 177 & $<0.001$ \\
\hline & $>37$ & 13 & 834 & \\
\hline
\end{tabular}

Note: ${ }^{1} 1004$ patients had size information. 


\section{Table 2}

Hazard ratios (HR) and $\mathrm{P}$ value for death associated with demographic and prognostic factors in patients with advanced pancreatic cancer by multivariate analyses.

\begin{tabular}{lcc}
\hline Characteristics $^{1}$ & HR & $P$ value \\
\hline Age $>60 \mathrm{yr}$ & 1.10 & 0.237 \\
Female & 0.95 & 0.517 \\
Head & 0.81 & 0.013 \\
Size $>4 \mathrm{~cm}$ & 1.22 & 0.015 \\
ECOG 0-1 & & 0.025 \\
Stage IV & 0.76 & $<0.001$ \\
Chemotherapy & 1.91 & $<0.001$ \\
Radiotherapy & 0.61 & 0.012 \\
CA19-9-Low\&Lewis $(+)$ & 0.69 & $<0.001$ \\
\hline
\end{tabular}

Note: ${ }^{1} 1039$ cases $;{ }^{2}$ Eastern Cooperative Oncology Group (ECOG) performance status, 0-1 vs. 2-3. 


\section{Table 3}

Molecular features of pancreatic cancer with the CA19-9-Low\&Lewis (+) and non CA19-9-Low\&Lewis (+) subgroups.

\begin{tabular}{llccc}
\hline Characteristics & Items & $\begin{array}{c}\text { CA19-9-Low } \\
\text { \&Lewis (+) }\end{array}$ & $\begin{array}{c}\text { non } \\
\text { CA19-9-Low\& } \\
\text { Lewis (+) }\end{array}$ & $\begin{array}{c}P \\
\text { value }\end{array}$ \\
\hline \multirow{2}{*}{ TP53 } & Positive & 39 & 202 & 0.317 \\
& Negative & 27 & 106 & \\
SMAD4 & Positive & 6 & 26 & 0.892 \\
& Negative & 25 & 116 & \\
CDKN2A & Positive & 9 & 38 & 0.797 \\
EGFR & Negative & 22 & 104 & \\
& Positive & 24 & 154 & 0.047 \\
HER2 & Negative & 40 & 147 & \\
& Positive & 40 & 177 & 0.659 \\
\hline
\end{tabular}


A

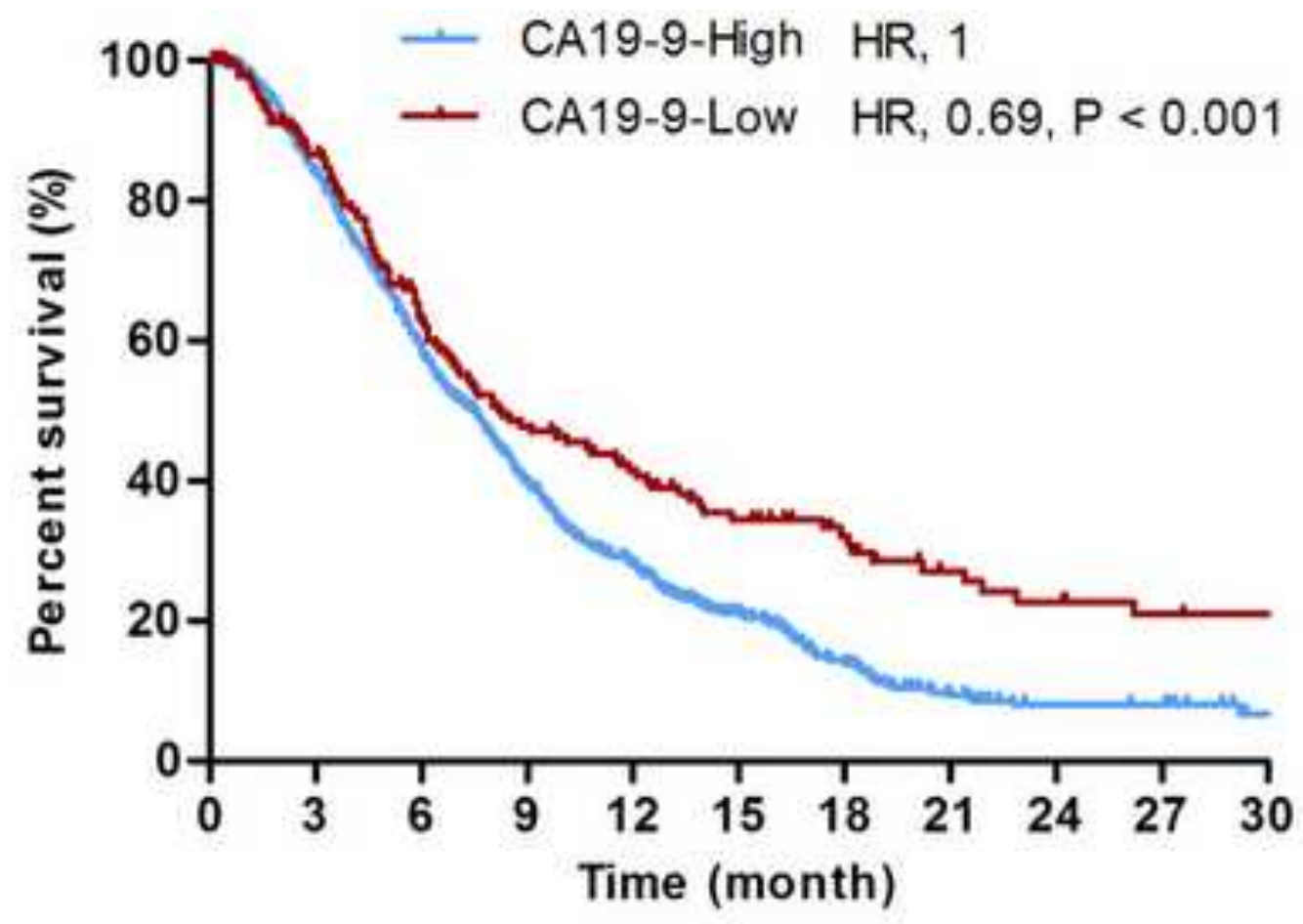

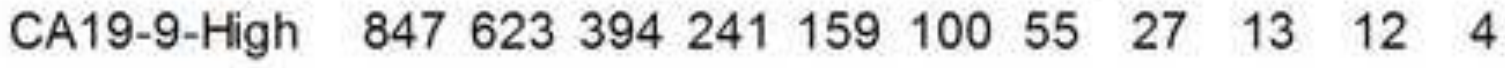
CA19-9-Low $192 \begin{array}{llllllllll}148 & 94 & 63 & 51 & 37 & 27 & 19 & 15 & 12 & 11\end{array}$

B

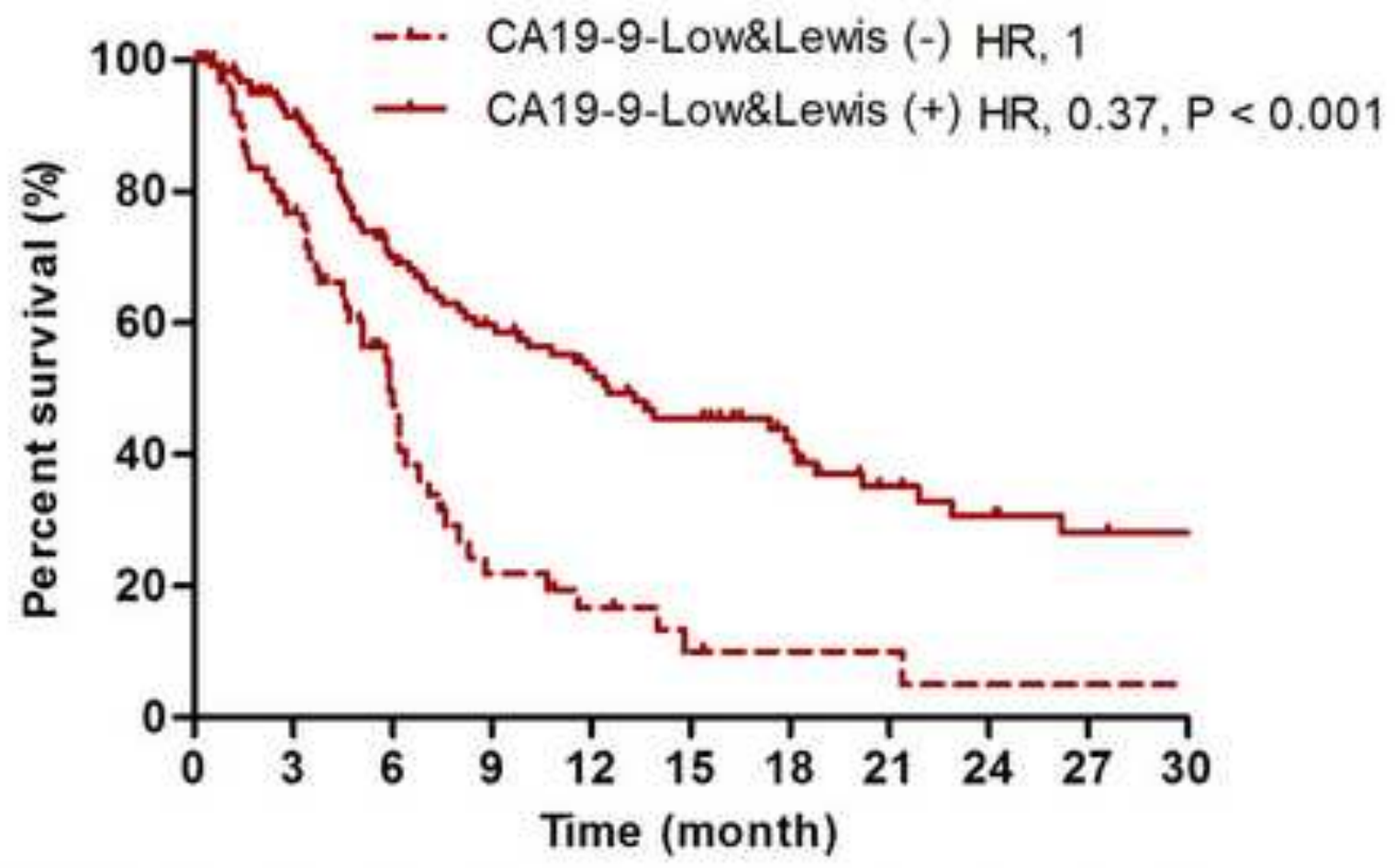

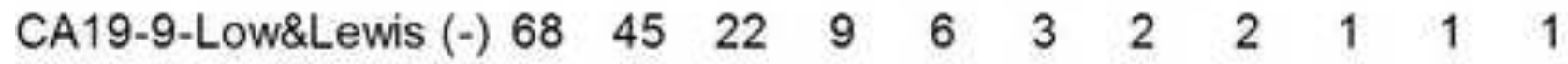
CA19-9-Low\&Lewis (+) $124 \begin{array}{llllllllll}103 & 72 & 54 & 45 & 34 & 25 & 17 & 14 & 11 & 10\end{array}$ 
-- Chemo.

- Chemo. + Radio.
$H R, 0.56, P<0.001$

$H R, 0.40, P<0.001$

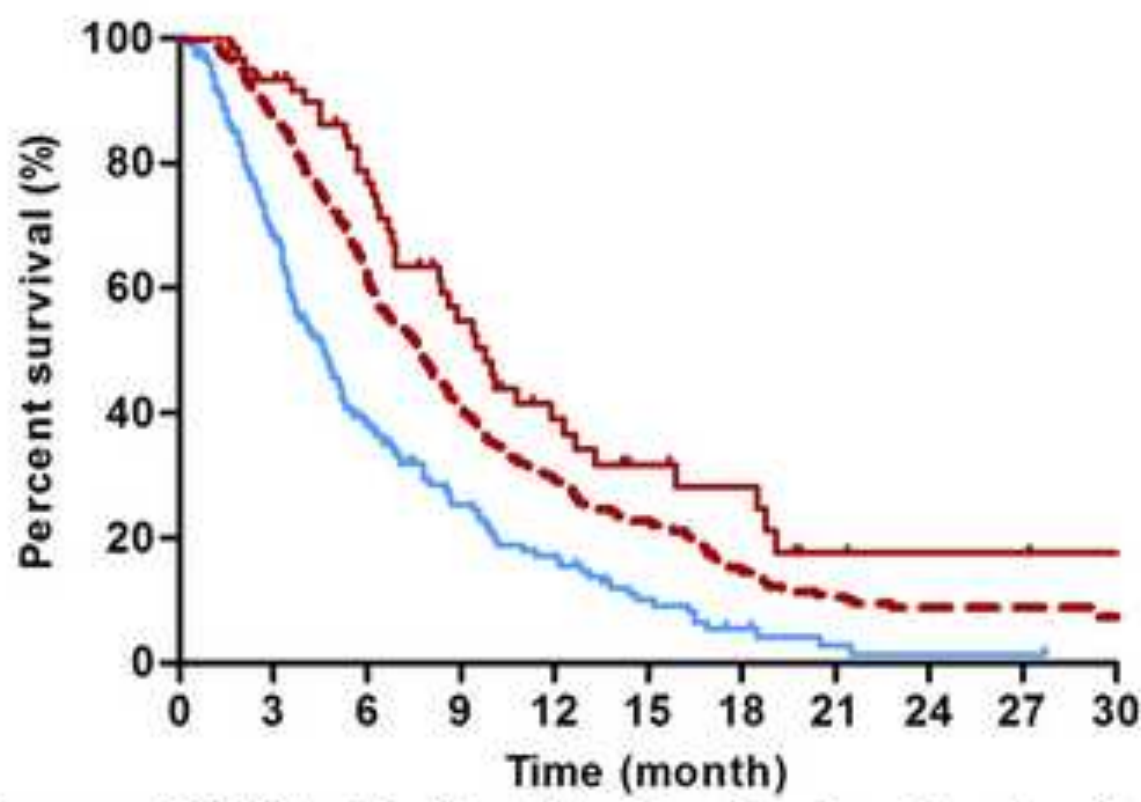

Best supportive care $\begin{array}{lllllllllll}198 & 104 & 53 & 31 & 21 & 11 & 5 & 2 & 1 & 1 & 0\end{array}$

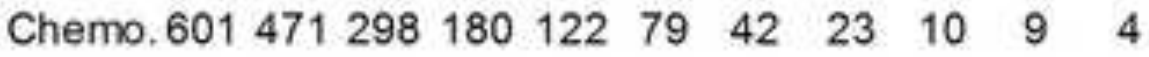
Chemo. + Radio. $\begin{array}{lllllllllll}61 & 55 & 42 & 25 & 16 & 10 & 8 & 3 & 2 & 2 & 1\end{array}$

B

- Best supportive care HR, 1

-- Chemo.

$H R, 0.80, P=0.444$

- Chemo. + Radio. HR, 0.30, P $=0.008$

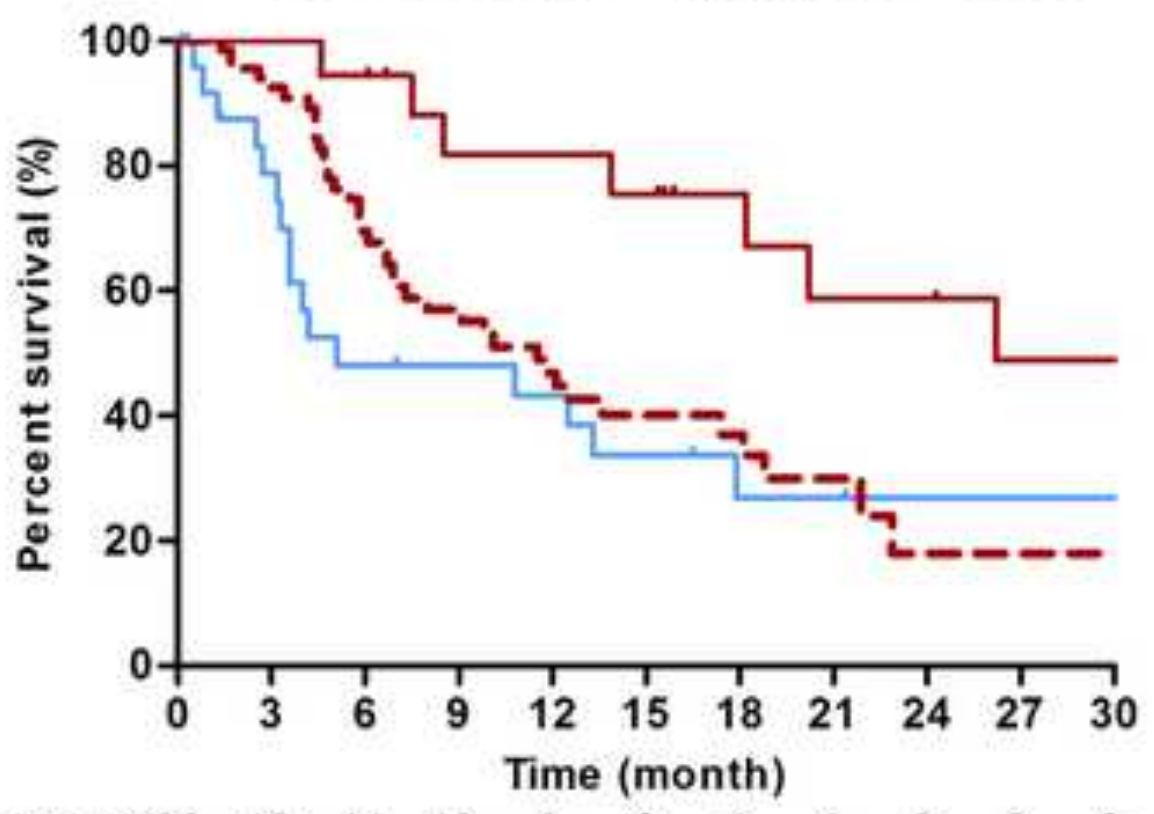

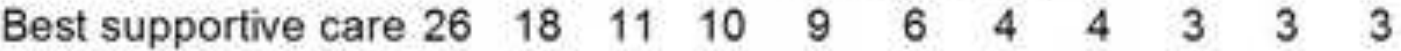

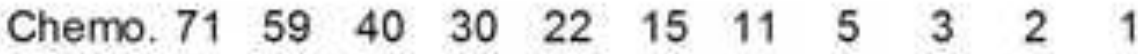
Chemo, + Radio. $\begin{array}{lllllllllll}18 & 18 & 17 & 13 & 13 & 12 & 9 & 7 & 7 & 5 & 5\end{array}$ 